\title{
Reconfigurable electronic circuits for magnetic fields controlled by structured light
}

\author{
K. Jana, K. R. Herperger $\odot$, F. Kong, Y. Mi $\odot$, C. Zhang, P. B. Corkum and S. Sederberg $₫ \bowtie$
}

Dynamic control over the conduction band electrons of a semiconductor is a central technological pursuit. Beyond electronic circuitry, flexible control over the spatial and temporal character of semiconductor currents enables precise spatiotemporal structuring of magnetic fields. Despite their importance in science and technology, the control of magnetic fields at the micrometre spatial scale and femtosecond temporal scale using conventional electromagnets remains challenging. Here, we apply structured light beams to interfering photoexcitation pathways in gallium arsenide to sculpt the spatial and momentum configuration of its conduction band population. Programmable control over several hundred micrometre-scale current elements is achieved by manipulating the wavefronts of an optical beam using a spatial light modulator, enabling vast flexibility in the excited current patterns. Using this platform, we demonstrate dynamic optoelectronic interconnects, circuits for spatially tailored magnetic fields and magnetic field lattices.

patial and temporal control over current flowing through semiconductors forms the basis of modern electronics and information processing. In metal-oxide-semiconductor circuits, current control arises from electric fields confined to the surfaces of fixed metallic features. State-of-the-art lithography enables three-dimensional routing of electrons at the nanometre scale, but the direction of each local drift current element is pre-determined by the orientation of the wire or electrode at that point in space. Moreover, the loss and capacitance of conventional electric interconnects limit the rate at which currents can be manipulated to the order of several gigahertz.

Alternatively, currents can be excited optically via quantum interference in electronic transitions ${ }^{1-3}$, photoconductive switching $^{4,5}$, the photovoltaic effect ${ }^{6,7}$, shift currents ${ }^{8}$ or strong-field photoexcitation processes ${ }^{9-11}$, enabling optoelectronic control at terahertz to petahertz rates. An enticing technological question that remains unexplored is whether electronic components-or even entire circuits-can be dynamically introduced into a semiconductor through optical excitation, making them independent of lithographically defined structures ${ }^{12}$. Such flexibility would enable external manipulation or optimization of a circuit's function and could relieve longstanding technological challenges such as the charging time of conventional wires.

Quantum interference in electronic transitions in a semiconductor using visible to mid-infrared light is a conceptually simple, yet powerful approach to current control. When a semiconductor is irradiated by an optical pulse, the crystal momentum-dependent transition rate from the valence band to the conduction band depends jointly on the electronic band structure and the time-varying electric-field excitation. Bichromatic or broadband light can introduce two or more parallel transition pathways at certain crystal momenta, enabling quantum interference, a form of coherent control ${ }^{1-3,13}$. Modern optical technology provides vast flexibility in the spatial, vectorial and temporal character of light ${ }^{14-19}$. A natural extension of conventional coherent control lies in applying spatially structured laser modes to a semiconductor to control the spatial arrangement of the excited currents. Recently, we have demonstrated spatio-vectorially resolved coherent control using cylindrical vector beams ${ }^{20}$. Current patterns resembling the electric fields of optical modes were excited and measured, but the availability of only a fixed mode prevented any flexibility in the current arrangement. Dynamic control of intricate current patterns would require contributions from many spatial modes, or spatial modulation of the light.

Semiconductor fabrication techniques have also been used to define optical displays, where the active material is chosen to allow an electric field full control over the amplitude, phase and polarization of the emitted light. Recently, optical display technology has been adapted for spatial modulation of the laser beams used for optical communications, enabling classical or quantum information to be carried on their mode ${ }^{21,22}$. In this Article, we convert information encoded onto the wavefront of an optical beam into a current arrangement in a semiconductor. We will show that, by controlling the phase and polarization of one of the components of a bichromatic field, display technology offers complete freedom to determine the current direction, pixel by pixel, in a one-to-one mapping between direction and phase delay.

We achieve current control in a semiconductor through the simultaneous excitation of a two-photon transition by an electric field oscillating at angular frequency $\omega$ and a single-photon transition by its second-harmonic $(2 \omega)$ using the relative phase between the two light waves, $\Delta \varphi_{\omega, 2 \omega}=2 \varphi_{\omega}-\varphi_{2 \omega}$. For a direct-bandgap semiconductor, the current density dynamics arising from electrons, $\mathbf{J}_{\mathrm{e}}$, or holes, $\mathbf{J}_{\mathrm{h}}$, can be described by

$$
\dot{\mathrm{J}}_{\mathrm{e}, \mathrm{h}}=\hat{\eta}_{\mathrm{e}, \mathrm{h}}: \mathbf{E}_{\omega} \mathbf{E}_{\omega} \mathbf{E}_{-2 \omega}+\text { c.c. }+\frac{\mathbf{J}_{\mathrm{e}, \mathrm{h}}}{\tau_{\mathrm{e}, \mathrm{h}}}
$$

where $\hat{\eta}_{\mathrm{e}, \mathrm{h}}$ is a fourth-rank tensor specific to the semiconductor, $\tau_{e, h}$ is the current relaxation timescale and $\mathbf{E}_{\omega}$ and $\mathbf{E}_{2 \omega}$ are the complex-valued vector amplitudes of the $\omega$ and $2 \omega$ fields, respectively ${ }^{3}$. The phase dependency of the current density injection rate becomes explicit when the fields and current are oriented along a common axis: 


$$
\dot{J}\left(\Delta \varphi_{\omega, 2 \omega}\right)=2 \eta_{0}\left(E_{\omega}\right)^{2} E_{2 \omega} \sin \left(\Delta \varphi_{\omega, 2 \omega}\right)
$$

where $\eta_{0}$ is a scalar constant related to $\hat{\eta}_{\mathrm{e}, \mathrm{h}}$. Evidently, the amplitude and sign of the current density can be controlled using $\Delta \varphi_{\omega, 2 \omega}$.

Full directional control of an injected current arises when quantum interference is driven with at least one circularly polarized light beam. Circular polarization can be equivalently represented by its $x$ - and $y$-electric-field content:

$$
E(t)=E_{0}\left(\widehat{\mathbf{x}} \cos \left(\omega t+\varphi_{0}\right) \pm \widehat{\mathbf{y}} \sin \left(\omega t+\varphi_{0}\right)\right)
$$

where $E_{0}$ is the electric field amplitude, $\varphi_{0}$ is a constant phase offset and $\widehat{\mathbf{x}}$ and $\widehat{\mathbf{y}}$ are unit vectors pointing in the $x$ and $y$ directions, respectively. The orthogonality of the $x$ - and $y$-electric-field components in space and time provides a conceptually simple means to control the transverse direction of the injected current using $\Delta \varphi_{\omega, 2 \omega}\left(\right.$ ref. $\left.^{23}\right)$ :

$$
\mathbf{J} \propto \widehat{\mathbf{x}} \sin \left(\Delta \varphi_{\omega, 2 \omega}\right) \pm \widehat{\mathbf{y}} \cos \left(\Delta \varphi_{\omega, 2 \omega}\right)
$$

Equation (4) shows that the current's direction depends on $\Delta \varphi_{\omega, 2 \omega}$ and, as in the case of linearly polarized excitation, its amplitude can be controlled using the electric-field amplitudes of the two beams. The inherently microscopic current injection mechanism and its independence from a predefined electric circuit are key elements that enable reconfigurable optoelectronic circuitry. Control over $\Delta \varphi_{\omega, 2 \omega}$ in a pixelated array, that is, $\Delta \varphi_{\omega, 2 \omega}\left(x_{n}, y_{n}\right)$, would enable each local phase pixel to be mapped to a current density pixel, $\mathbf{J}\left(x_{m}, y_{m}\right)$, via equation (4). The collective current driven by the ensemble of pixels would embody an optoelectronic circuit.

To realize this functionality, we only require two Gaussian laser beams with femtosecond pulse durations and a spatial light modulator (SLM), arranged in the experimental configuration presented in Fig. 1a (additional details are provided in the Methods). A laser beam at $\lambda_{\omega}=1,480 \mathrm{~nm}$ and its second harmonic at $\lambda_{2 \omega}=740 \mathrm{~nm}$ are used to inject currents into the conduction band of low-temperature gallium arsenide (LT-GaAs). The bandgap energy of the LT-GaAs is $E_{\mathrm{g}}=1.44 \mathrm{eV}$, such that direct transitions are excited by the $2 \omega$ beam and two-photon transitions are driven by the $\omega$ beam.

Before irradiating the semiconductor, the $\omega$ and $2 \omega$ pulses are split into separate optical paths, and two independent contributions to $\Delta \varphi_{\omega, 2 \omega}\left(x_{n}, y_{n}\right)$ are introduced: the first is a global relative phase, $\Delta \varphi_{\text {global }}$, defined by a piezoelectric-controlled optical delay. This delay controls the temporal overlap of the two pulses and sets a constant phase offset between them (Supplementary Note 1). Second, to enable programmable control of sub-beam-diameter elements comprising the phase fronts between the two beams, the $2 \omega$ beam is reflected from an SLM, introducing a pixelated relative phase, $\Delta \varphi_{\text {SLM }}\left(x_{n}, y_{n}\right)$. Each pixel of the combined relative phase, $\Delta \varphi_{\omega, 2 \omega}\left(x_{n}, y_{n}\right)=\Delta \varphi_{\text {global }}+\Delta \varphi_{\mathrm{SLM}}\left(x_{n}, y_{n}\right)$, enables independent coherent control of a current element (Methods). In addition, the $\omega$ beam is transmitted through a quarter-wave plate to obtain circular polarization. After combining the two beams collinearly, $\Delta \varphi_{\text {SLM }}\left(x_{n}, y_{n}\right)$ is de-magnified fivefold and imaged onto the plane of the LT-GaAs detector, which has an active area with dimensions of $25 \mu \mathrm{m} \times 25 \mu \mathrm{m}$ (Supplementary Note 2 ). An artistic rendering of the two beams, their relative phase arrangement $\left(\Delta \varphi_{\omega, 2 \omega}\left(x_{n}, y_{n}\right)\right)$ and the resulting current pattern is shown in Fig. $1 \mathrm{~b}$.

We measured the dependence of the $x$ and $y$ currents, $I_{x}$ and $I_{y}$, respectively, on $\Delta \varphi_{\text {global }}$ in a single $25 \mu \mathrm{m} \times 25 \mu \mathrm{m}$ detection pixel. As shown in Fig. $1 c, I_{x}$ and $I_{y}$ oscillate $\pi / 2$ out of phase as $\Delta \varphi_{\text {global }}$ is adjusted. The time-averaged current we measured is $\sim 1 \mathrm{pA}$, corresponding to roughly 6,242 detected electrons per laser pulse. From the current relaxation timescale $\left(\tau_{\mathrm{r}}=250 \mathrm{fs}\right)$ and the detector dimensions, we estimated the peak current density to be $1 \times 10^{8} \mathrm{~A} \mathrm{~m}^{-2}$.
Plotting $I_{x}$ and $I_{y}$ parametrically versus $\Delta \varphi_{\text {global }}$ over $2 \pi$ (grey-shaded area, Fig. 1c) illustrates that current of constant amplitude, but adjustable direction, is injected (Fig. 1d). This single pixel serves as a building block for general optoelectronic circuits.

To extend this control to pixel arrays, we introduced wire-like features resembling conventional electronic circuits to $\Delta \varphi_{\text {SLM }}\left(x_{n}\right.$, $\left.y_{n}\right)$, as depicted in Fig. $2 \mathrm{a}-\mathrm{c}$. We envisaged a single, non-existent electrode on the left-hand side, which can be connected to one of three electrodes on the right-hand side. The current patterns that we excite serve as 'optoelectronic interconnects' between different pairs of electrodes, controlling the function of a non-existent, underlying electronic circuit.

Routing current in the $+x,-x,+y$ or $-y$ directions can be achieved by setting $\Delta \varphi_{\text {SLM }}\left(x_{n}, y_{n}\right)$ in the appropriate pixels to $0, \pi, 3 \pi / 2$ or $\pi / 2 \mathrm{rad}$, respectively. The SLM phase patterns used to drive the interconnections illustrated in the top row of Fig. 2 are presented in the corresponding panels in the middle row. A chequerboard background pattern is superimposed on $\Delta \varphi_{\text {SLM }}\left(x_{n}, y_{n}\right)$ to eliminate current from regions other than those occupied by the optoelectronic interconnects. These rapid phase variations prevent light from being properly imaged onto the detector, selectively suppressing current injection.

Leaving $\Delta \varphi_{\omega, 2 \omega}\left(x_{n}, y_{n}\right)$ fixed, the sub-beam-diameter current detector is raster-scanned across the exciting beams in a square grid with $25-\mu \mathrm{m}$ steps in the $x$ and $y$ directions to obtain a complete spatial mapping of the current vector excited by the two laser pulses. Raw data for the $I_{x}$ and $I_{y}$ current components are plotted in Supplementary Note 3. The spatial arrangements of the transverse current vector obtained using the SLM patterns in Fig. $2 \mathrm{~d}-\mathrm{f}$ are shown in Fig. 2g-i, respectively. Independent control over both $I_{x}$ and $I_{y}$ in wire-like features connecting the imaginary electrodes is clearly demonstrated. The ultimate spatial resolution of these connections is governed by the light wave diffraction limit and therefore holds the potential for high-density, optically defined electric circuits.

Motivated by the fundamental relationship between currents and magnetic fields, and the present limitations in magnetic field technology, we next focused on demonstrating flexible current arrangements for generating magnetic fields. Magnetic fields are integral to the picometre spatial resolution of electron microscopes ${ }^{24,25}$, the operation of free-electron lasers ${ }^{26,27}$, spintronic devices ${ }^{28,29}$, magnetic resonance microscopy ${ }^{30}$, magnetic traps for cold atoms ${ }^{31}$ and magnetic skyrmions ${ }^{32}$. The availability of optically synchronized, subpicosecond magnetic-field impulses would provide the possibility to apply time-varying magnetic fields to instruments, materials or devices, and to visualize ultrafast magnetic-field-induced dynamics in nature. Simultaneously, the spatial arrangement of these magnetic fields could be controlled and optimized on dimensions approaching the light wave diffraction limit.

Reconfiguring the SLM-defined wires to drive currents around a square-shaped loop excites a longitudinal magnetic field at its centre. The SLM pattern used to inject such a loop current is shown in Fig. 3a, and the measured current vector arrangement is plotted in Fig. 3b (raw data are plotted in Supplementary Note 4). Knowing the spatial arrangement of current elements and estimating their excitation and relaxation dynamics enables us to calculate the spatiotemporal magnetic fields using retarded potentials (Supplementary Note 6). A snapshot of the longitudinal magnetic field calculated $200 \mathrm{fs}$ after excitation is shown in Fig. 3c.

We reconfigured this magnetic field by introducing a conical gradient phase pattern to the SLM (Fig. $3 \mathrm{~d}$ ). This pattern delays the phase front of the $2 \omega$ beam by $2 \pi$ as the azimuthal coordinate is traversed, adding orbital angular momentum (OAM) of $\hbar(m=1$, where $m$ is the number of units of OAM) to the beam. When imaged onto the detector, this $2 \pi$ phase delay is converted into a $2 \pi$ rotation of the current vector, driving a ring current that occupies the entire 
a

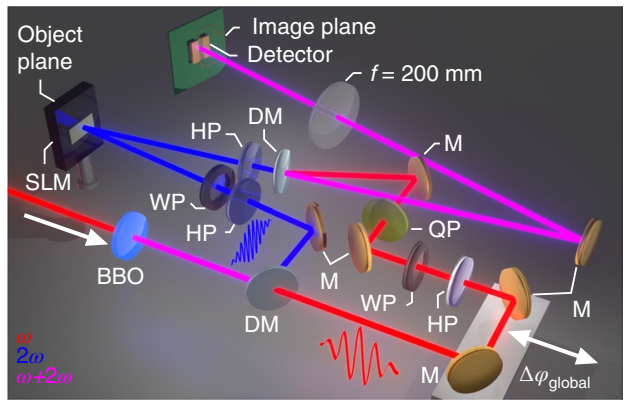

c

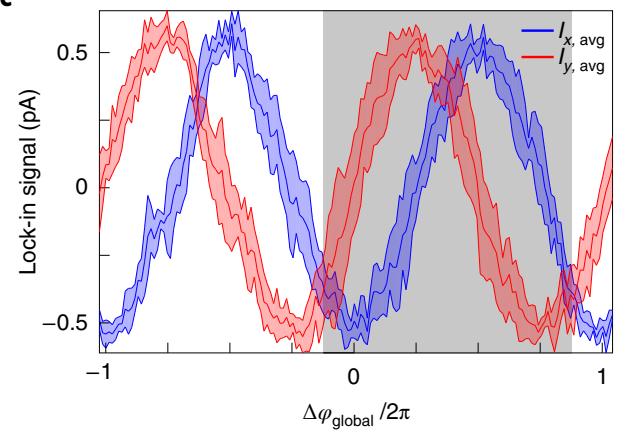

b

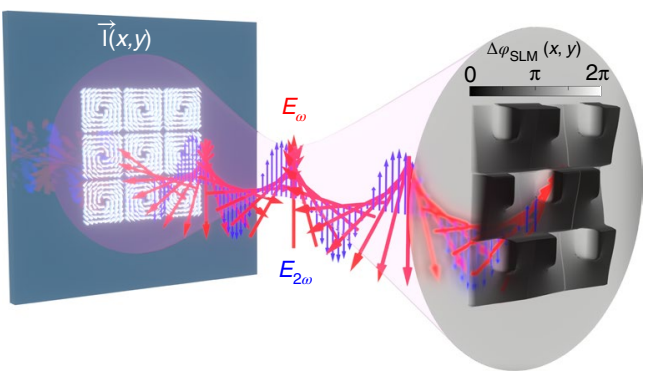

d

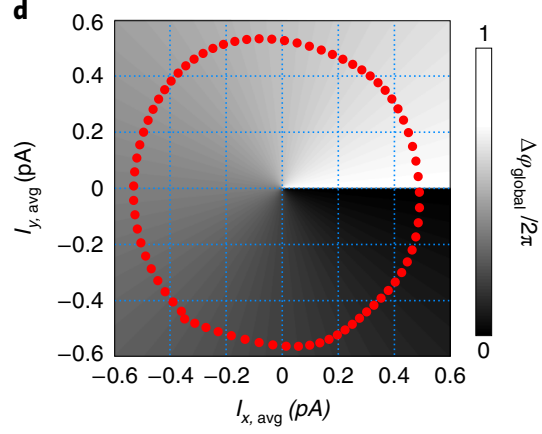

Fig. 1 | Transverse vectorial control of current pixels. a, Schematic of the experimental configuration. Two laser beams are imaged collinearly onto the LT-GaAs optoelectronic detector. The first beam, centred at $\lambda_{\omega}=1,480 \mathrm{~nm}$, is circularly polarized. The second beam $\left(\lambda_{2 \omega}=740 \mathrm{~nm}\right)$ is reflected from an SLM, where a pixelated phase is imprinted onto the wavefront. $M$, metallic mirror; DM, dichroic mirror; QP, quarter-wave plate; HP, half-wave plate; WP, wire-grid polarizer; SLM, spatial light modulator; BBO, $\beta$-barium borate crystal. $\mathbf{b}$, Artistic rendering of the circularly polarized $\omega$ beam, the linearly polarized $2 \omega$ beam and the relative phase between their wavefronts, $\Delta \phi_{\omega, 2 \omega}(x, y)$. When incident on the semiconductor, the relative phase, controlled at each pixel of the SLM by the delay between the $\omega$ and $2 \omega$ pulses, determines the direction of the resulting current element in the semiconductor.

c, Measurement of the $x$ (blue) and $y$ (red) current components in one detection pixel as the global relative phase, $\Delta \phi_{\text {global }}$, is varied over two cycles of the $2 \omega$ beam. The shaded blue and red regions represent the $1 \sigma$ confidence interval of the measured data. $\mathbf{d}$, Parametric plot of the data from $\mathbf{c}$ over one optical cycle (shown by the grey-shaded region of $\mathbf{c}$ ), demonstrating that $\Delta \phi_{\omega, 2 \omega}(x, y)$ can be used to precisely control the transverse direction of currents. Fourier domain filtering is applied to the data of $\mathbf{c}$ to obtain this plot.

beam radius (Fig. 3e). This produces a large, round magnetic field in the plane of the detector (Fig. 3f).

Impressing $m=3$ onto the $2 \omega$ beam (Fig. $3 \mathrm{~g}$ ) introduces four individual loop currents, each localized to a quadrant of the exciting beam (Fig. 3h). The corresponding four-lobed magnetic field distribution is shown in Fig. 3i. More generally, a beam with $m>1$ will introduce $2(m-1)$ loop currents and magnetic field lobes. Although we do not study it here, spin-polarized currents will arise due to angular momentum conservation.

Radial modulation was added to the magnetic field distribution using a spiral phase pattern (Fig. 3j). Although distinct loop currents are not observed in the resulting current distribution (Fig. 3k), a net circulating current with direction that alternates with increasing radial coordinate produces a collection of concentric magnetic field rings with alternating sign (Fig. 31). The radial periodicity of this magnetic field could make it useful as a phase grating for electron beams or for chip-scale free-electron lasers. In each of these examples, the 180-fs current relaxation timescale in the LT-GaAs detector produces similarly short magnetic-field transients ${ }^{33,34}$. By applying numerical estimates of the peak current density to these current distributions, we estimated the amplitude of the excited magnetic fields to be on the order of $0.05-1 \mathrm{mT}$ (ref. ${ }^{3}$ ). We envisage the application of these magnetic impulses to understanding the ultrafast magnetic-field-induced response of materials ${ }^{35}$.

To demonstrate extreme spatial tailoring of magnetic fields, we created lattices of ring currents, where the magnetic field's amplitude and sign at each lattice site is adjusted by controlling the amplitude and direction of the local ring current. Such lattices could be used for all-optical control of magnetic memory arrays, as a dynamic magnetic metamaterial, for quantum simulation using ultracold atoms, for manipulation of the optoelectronic properties of quantum dot arrays or to excite impulsive, chip-scale undulators or wigglers for free-electron lasers.

As a proof of principle, we excited $3 \times 3$ lattices of ring currents, with larger arrays possible using different experimental configurations. In the first SLM pattern (Fig. 4a), we introduced a $3 \times 3$ lattice of counterclockwise conical gradients. Current circulation at each lattice site was observed in the measured current arrangements (Fig. 4b; raw data are plotted in Supplementary Note 5). The calculated magnetic field lattice is shown in Fig. 4c. In addition to the primary magnetic fields at each lattice site, magnetic fields with opposite sign are present at the interstitial sites due to an effective ring current formed by the corners of the four adjacent sites.

Next, the conical gradient direction at each lattice site was set to be opposite that of its adjacent sites (Fig. 4d). The alternating direction of current circulation is clearly observed in the measured current pattern (Fig. 4e), which naturally leads to alternating magnetic field directions in the lattice (Fig. 4f).

Finally, we introduced a 'defect' to the centre site of the lattice. Radial currents are orthogonal to the ring currents and do not produce a longitudinal magnetic field. Therefore, a conical gradient rotated by $\pi / 2$ was introduced to the centre lattice site, as illustrated in Fig. 4g. Loop currents were measured at each of the edge lattice sites, and a distinct radial current is observed at the centre 

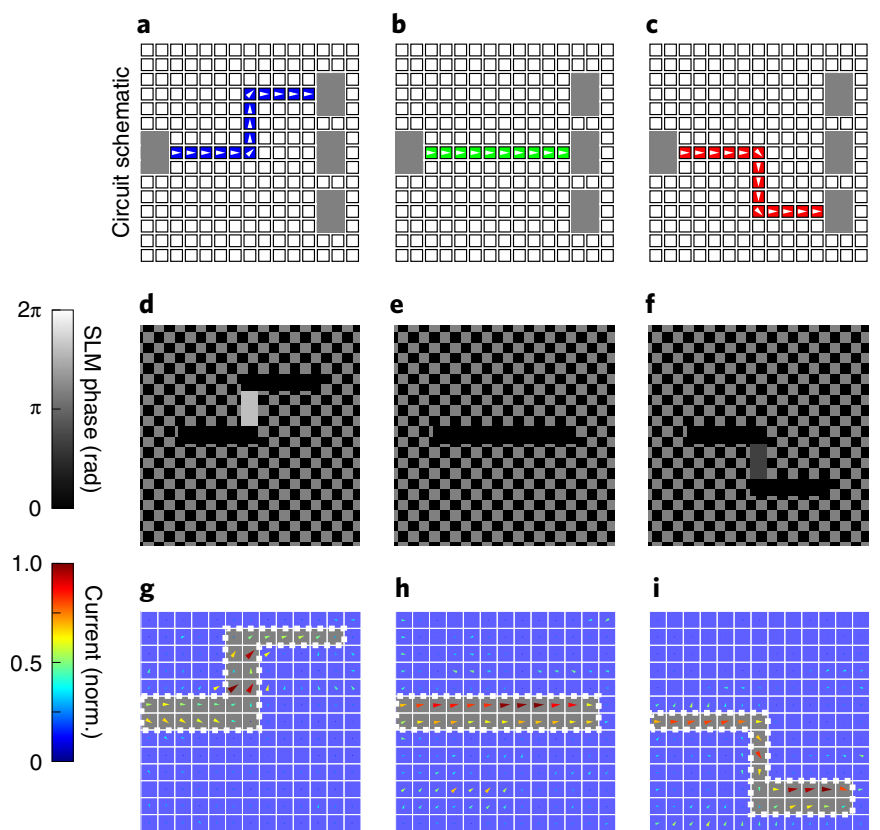

h

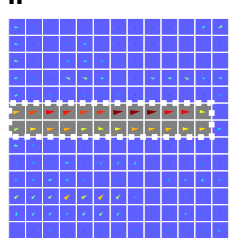

i

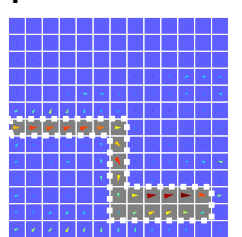

Fig. 2 | Reconfigurable optoelectronic interconnects. a-c, Schematics of three optoelectronic circuits that can be excited either separately or simultaneously, resulting in currents being driven between different pairs of fictive electrodes 'contacted' to a non-existent integrated circuit. d-f, The SLM phase patterns used to control the direction of currents in the optoelectronic interconnects in a-c are shown in $\mathbf{d}$, e and $\mathbf{f}$, respectively. The chequerboard pattern in the background is used to introduce frequent phase discontinuities to the beam. This high-order spatial content does not image properly onto the detector and, as a result, well-defined currents are not excited in these regions. $\mathbf{g}-\mathbf{i}$, The measured current vector arrangements produced when the SLM patterns in $\mathbf{d}$, e and $\mathbf{f}$ are applied ( $\mathbf{g}, \mathbf{h}$ and $\mathbf{i}$, respectively). The size and colour of each arrowhead represent the amplitude of the local current. The white dotted outlines represent the region where current is expected.

site (Fig. 4h). The resulting magnetic field snapshot is shown in Fig. 4i, where the magnetic field is effectively suppressed at the centre site. Notably, current contributions from the four nearest-neighbour lattice sites produce a net counterclockwise ring current around the centre site, resulting in a positive magnetic field that arrives $\sim 100$ fs later.

In summary, we have introduced a method to map the phase difference between a fundamental and a second harmonic beam, one to one, onto the direction of a semiconductor current, providing $360^{\circ}$ control over its direction. A direct outcome of this method will be an all-solid-state phase detector that will simplify two-colour interferometry. Using a one-dimensional realization of this detector, we have demonstrated reconfigurable optoelectronic circuitry using a semiconductor material platform and technologically important light wavelengths. In contrast to metallic conductors, which introduce loss and latency to electrical signals, these currents can be turned on within the timescale of our femtosecond laser pulses and current is introduced to the entire circuit simultaneously. The multi-kilohertz refresh rate of modern SLMs enables the function of the circuit to be changed at a similar rate, a feature that could be used to enhance the versatility and security of electronic circuits.

The availability of mega-pixel SLMs provides unprecedented degrees of freedom for manipulating and optimizing magnetic fields. We envisage the application of optically driven magnetic fields to the adaptive control of charged-particle beam collimation and focusing, chip-scale free-electron lasers and high-bandwidth

optical writing to magnetic memory devices. The impulsive character of these magnetic fields will make them unique probes of the ultimate bandwidth limits of magnetic and spintronic technologies. In addition, this optically controlled circuitry can be shrunk to approximately the dimensions of the second harmonic wavelength, enabling control over similarly compact magnetic fields. Transferring this scheme to large-bandgap dielectrics and ultraviolet wavelengths could enable manipulation of magnetic fields with $100-\mathrm{nm}$ dimensions on sub-femtosecond timescales. Although experimentally challenging, we envision that optical excitation of nanometre-scale magnetic fields would in turn enable excitation and control of individual skyrmions.

We note that technological applications of this scheme would benefit from a reduction in the laser power requirements and the use of materials with a large $\chi^{(3)}$ coefficient. Although transient currents are desirable in many applications, others benefit from long-lived currents. Extending this scheme to other quantum interference effects and emerging materials exhibiting strong optical absorption and coherent electron transfer over exceptional length scales will broaden the scope of application of these results. In particular,
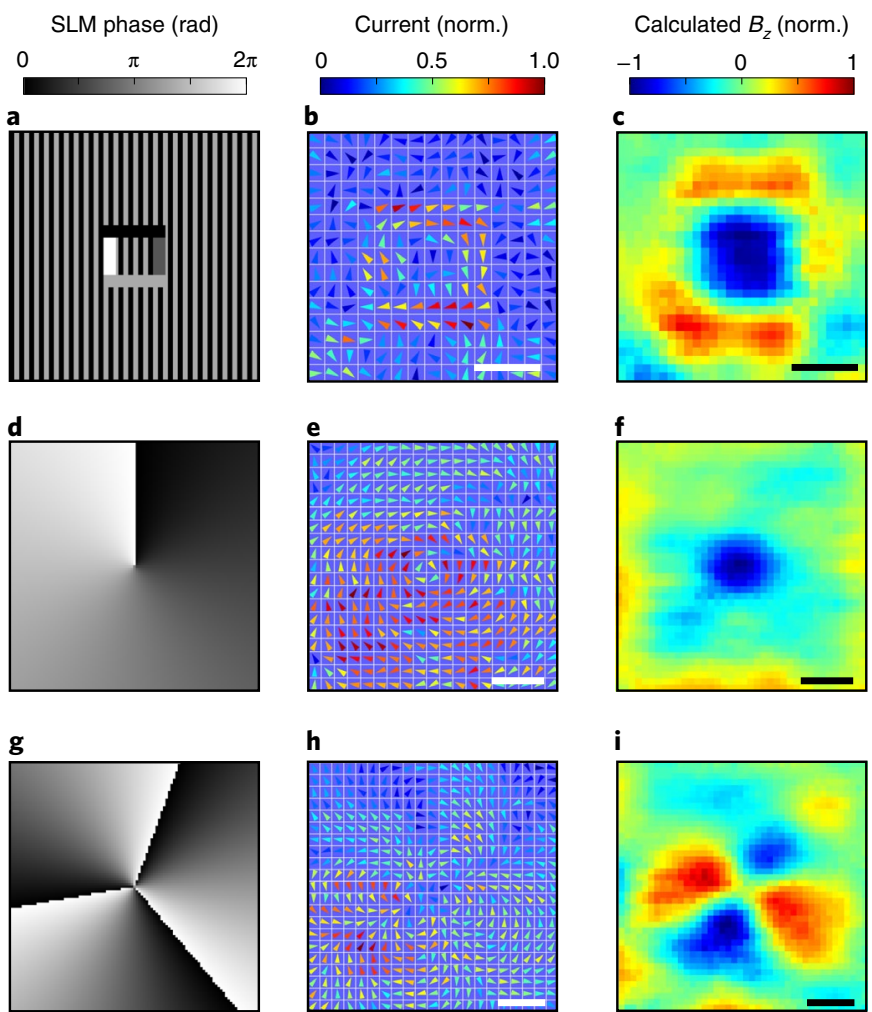

h
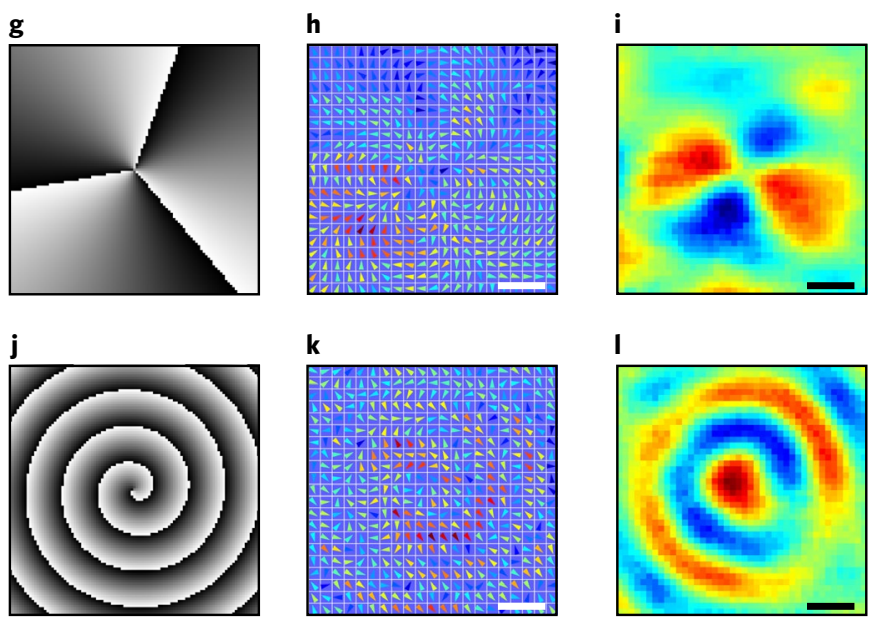

Fig. 3 | Spatially tailored magnetic fields. a,b,d,e,g,h,j,k, The SLM phase patterns shown in $\mathbf{a}, \mathbf{d}, \mathbf{g}$ and $\mathbf{j}$ are used to drive the current vector arrangements shown in $\mathbf{b}, \mathbf{e}, \mathbf{h}$ and $\mathbf{k}$, respectively. Retarded potentials are used to calculate the resulting longitudinal magnetic field arrangement, $B_{z}(x, y)$, at an instant $t=200$ fs after current excitation. $\mathbf{c}, \mathbf{f}, \mathbf{i}, \mathbf{I}$, , The magnetic field arrangements corresponding to the currents in $\mathbf{b}, \mathbf{e}, \mathbf{h}$ and $\mathbf{k}(\mathbf{c}, \mathbf{f}, \mathbf{i}$ and I, respectively). Scale bars, $100 \mu \mathrm{m}$. 

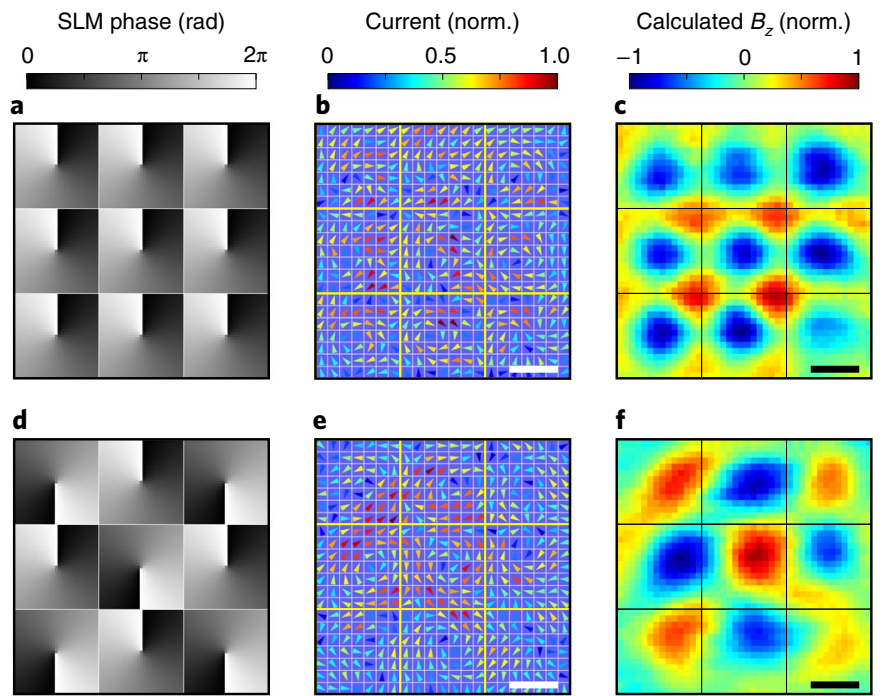

g

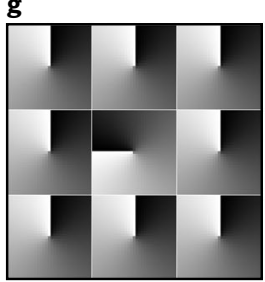

h
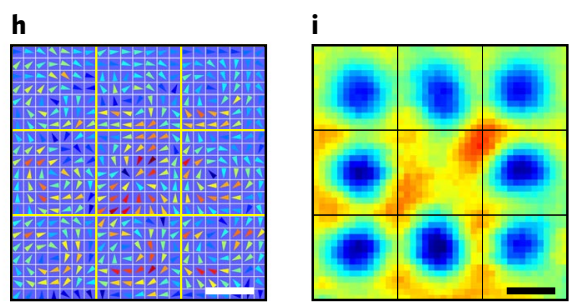

Fig. 4 | Magnetic field lattices. a,d,g, SLM phase patterns consisting of $3 \times 3$ arrays of conical gradients oriented to control the amplitude and sign of magnetic fields at each lattice site. $\mathbf{b}, \mathbf{e}, \mathbf{h}$, Applying the phase patterns in $\mathbf{a}, \mathbf{d}$ and $\mathbf{g}$ to the $2 \omega$ beam yields the current arrangements shown in $\mathbf{b}$, e and $\mathbf{h}$, respectively, where sub-beam diameter ring currents or radial currents are clearly resolved. $\mathbf{c}, \mathbf{f}, \mathbf{i}$, Snapshots of the resulting longitudinal magnetic fields $B_{z}$ corresponding to $\mathbf{a}, \mathbf{d}$ and $\mathbf{g}$ (c, $\mathbf{f}$ and $\mathbf{i}$, respectively) at an instant $t=200 \mathrm{fs}$ after current excitation. Scale bars, $100 \mu \mathrm{m}$.

quantum interference effects in monolayers such as graphene and transition metal dichalcogenides will be of future relevance.

Finally, we have demonstrated an approach to meta-optics that might be called active meta-optics. In our experiment, we do not need a material structure. Instead, we induce a current structure. Each current pixel comprising the active meta-surface serves as a source of electromagnetic radiation. Because we have used LT-GaAs, the current transient of each pixel will emit terahertz radiation and the collective terahertz radiation emitted by the entire pixel array can be shaped in any way that is consistent with the GaAs response and with Maxwell's equations.

\section{Online content}

Any methods, additional references, Nature Research reporting summaries, source data, extended data, supplementary information, acknowledgements, peer review information; details of author contributions and competing interests; and statements of data and code availability are available at https://doi.org/10.1038/ s41566-021-00832-9.

Received: 2 February 2021; Accepted: 21 May 2021;

Published online: 21 June 2021

\section{References}

1. Dupont, E., Corkum, P. B., Liu, H. C., Buchanan, M. \& Wasilewksi, Z. R. Phase-controlled currents in semiconductors. Phys. Rev. Lett. 74, 3596-3599 (1995).
2. Atanasov, R., Haché, A., Hughes, J. L. P., van Driel, H. M. \& Sipe, J. E. Coherent control of photocurrent injection in bulk semiconductors. Phys. Rev. Lett. 76, 1703-1706 (1996).

3. Haché, A., Sipe, J. E. \& van Driel, H. M. Quantum interference control of electrical currents in GaAs. IEEE J. Quantum Electron. 34, 1144-1154 (1998)

4. Auston, D. H. Picosecond optoelectronic switching and gating in silicon. Appl. Phys. Lett. 26, 1144-1154 (1998).

5. Auston, D. H. Ultrafast optoelectronics. Top. Appl. Phys. 60, 183-233 (1988).

6. Belinicher, V. I. \& Sturman, B. I. The photogalvanic effect in media lacking a center of symmetry. Sov. Phys. Usp. 23, 199-223 (1980).

7. Choi, T., Lee, S., Choi, Y. J., Kiryukhin, V. \& Cheong, S.-W. Switchable ferroelectric diode and photovoltaic effect in $\mathrm{BiFeO}_{3}$. Science 324, 63-66 (2009).

8. Côté, D., Laman, N. \& van Driel, H. M. Rectification and shift currents in GaAs. Appl. Phys. Lett. 80, 905-907 (2002).

9. Schiffrin, A. et al. Optical-field-induced current in dielectrics. Nature 493, 70-74 (2013).

10. Higuchi, T., Heide, C., Ullmann, K., Weber, H. B. \& Hommelhoff, P. Light-field-driven currents in graphene. Nature 550, 224-228 (2017).

11. Sederberg, S. et al. Attosecond optoelectronic field measurement in solids. Nat. Commun. 11, 430 (2020).

12. Forbes, A. Sculpting electric currents with structured light. Nat. Photon. 14, 656-657 (2020).

13. Shapiro, M. \& Brumer, P. Quantum Control of Molecular Processes (Wiley, 2012).

14. Rubinsztein-Dunlop, H. et al. Roadmap on structured light. J. Opt. 19, 013001 (2017).

15. Yu, N. \& Capasso, F. Flat optics with designer metasurfaces. Nat. Mater. 13, 139-150 (2014).

16. Zhan, Q. Cylindrical vector beams: from mathematical concepts to applications. Adv. Opt. Photon. 1, 1-57 (2009).

17. Forbes, A., de Oliveira, M. \& Dennis, M. R. Structured light. Nat. Photon. 15, 253-262 (2021).

18. Hassan, M. T. et al. Optical attosecond pulses and tracking the nonlinear response of bound electrons. Nature 530, 66-70 (2016).

19. Hammond, T. J., Villeneuve, D. M. \& Corkum, P. B. Producing and controlling half-cycle near-infrared electric-field transients. Optica 4, 826-830 (2017).

20. Sederberg, S. et al. Vectorized optoelectronic control and metrology in a semiconductor. Nat. Photon. 14, 680-685 (2020).

21. Fickler, R. et al. Quantum entanglement of high angular momenta. Science 338, 640-643 (2012).

22. Wang, J. et al. Terabit free-space data transmission employing orbital angular momentum multiplexing. Nat. Photon. 6, 488-496 (2012).

23. Bhat, R. D. R. \& Sipe, J. E. Optically injected spin currents in semiconductors. Phys. Rev. Lett. 85, 5432-5435 (2000).

24. Batson, P. E., Dellby, N. \& Krivanek, O. L. Sub-ångstrom resolution using aberration corrected electron optics. Nature 418, 617-620 (2002).

25. Erni, R., Rossell, M. D., Kisielowksi, C. \& Dahmen, U. Atomic-resolution imaging with a sub-50-pm electron probe. Phys. Rev. Lett. 102, 096101 (2009)

26. Kondratenko, A. M. \& Saldin, E. L. Generation of coherent radiation by a relativistic electron beam in an ondulator. Part. Accel. 10, 207-216 (1980).

27. Murphy, J. B. \& Pellegrini, C. Free electron lasers for the XUV spectral region. Nucl. Instrum. Methods Phys. Res. A 237, 159-167 (1985).

28. Fiederling, R. et al. Injection and detection of a spin-polarized current in light-emitting diode. Nature 402, 787-790 (1999).

29. Zutic, I., Fabian, J. \& Sarma, S. D. Spintronics: fundamentals and applications. Rev. Mod. Phys. 76, 323-410 (2004).

30. Rugar, D., Budakian, R., Mamin, H. J. \& Chui, B. W. Single spin detection by magnetic resonance force microscopy. Nature 430 , 329-332 (2004).

31. Pritchard, D. E. Cooling neutral atoms in a magnetic trap for precision spectroscopy. Phys. Rev. Lett. 51, 1336-1339 (1983).

32. Mühlbauer, S. et al. Skyrmion lattice in a chiral magnet. Science 323, 915-919 (2009).

33. Leitenstorfer, A., Fürst, C., Laubereau, A. \& Kaiser, W. Femtosecond carrier dynamics in GaAs far from equilibrium. Phys. Rev. Lett. 76, 1545-1548 (1996).

34. Sederberg, S., Kong, F. \& Corkum, P. B. Tesla-scale terahertz magnetic impulses. Phys. Rev. X 10, 011063 (2020).

35. Walowski, J. \& Münzenberg, M. Ultrafast magnetism and $\mathrm{THz}$ spintronics. J. Appl. Phys. 120, 140901 (2016).

Publisher's note Springer Nature remains neutral with regard to jurisdictional claims in published maps and institutional affiliations.

(C) The Author(s), under exclusive licence to Springer Nature Limited 2021 


\section{Methods}

Optical system. In the set-up, an optical parametric amplifier (Light Conversion, HE TOPAS) is pumped by amplified pulses from a Ti:sapphire laser system (Coherent, Legend Elite Duo; wavelength, $\lambda=800 \mathrm{~nm}$; repetition rate, $f_{\text {rep }}=1 \mathrm{kHz}$; pulse energy, $E_{\mathrm{p}}=5 \mathrm{~mJ}$; pulse duration, $\tau_{\mathrm{p}}=30 \mathrm{fs}$ ). The beam is spatially filtered by coupling it into a hollow-core capillary in ambient air. The transmitted mode is propagated through a $\beta$-barium borate crystal phase-matched for second-harmonic generation. A dichroic mirror is used to separate the second-harmonic $(2 \omega)$ beam from the fundamental $(\omega)$ beam. The energy of each resulting beam is controlled using a half-wave plate followed by a wire-grid polarizer. The $\omega$ beam is transmitted through a quarter-wave plate, converting its polarization state to circular. The $2 \omega$ beam is reflected from an SLM (Hamamatsu, LCOS-SLM) and a half-wave plate is used to control its polarization. The $\omega$ and $2 \omega$ beams are then combined collinearly using a second dichroic mirror. A $f=200 \mathrm{~mm}$ plano-convex lens is used to image and de-magnify the phase pattern introduced to the SLM onto the plane of the LT-GaAs detector. Typical peak intensities used for the $\omega$ and $2 \omega$ beams are $I_{\omega}^{\mathrm{p}}=90 \mathrm{GW} \mathrm{cm}^{-2}$ and $I_{2 \omega}^{\mathrm{p}}=0.28 \mathrm{GW} \mathrm{cm}^{-2}$.

Current detector. Two aluminium electrodes separated by a $25-\mu \mathrm{m}$ gap were patterned onto a LT-GaAs film. The semiconductor substrate was interfaced to a printed circuit board, which was connected to a lock-in amplifier (Stanford Research Systems SR830). An optical mask placed on the detector limited the detection aperture to a $25 \mu \mathrm{m} \times 25 \mu \mathrm{m}$ square. The strong directionality of the device to the excited currents enabled detection of the $x$ component of the current when the electrode gap was oriented along $\widehat{\mathbf{y}}$ and the $y$ component when the gap was aligned along $\widehat{\mathbf{x}}$.

Signal acquisition. The current detector was mounted to a motorized $x y$-translation stage assembly, which was raster-scanned in a $2 \mathrm{D}$ grid with $25-\mu \mathrm{m}$ step size. At each step, the current signal was acquired over a 500-ms time window using the lock-in amplifier.

\section{Data availability}

The raw data that support the findings of this study are available from the corresponding author upon reasonable request.

\section{Acknowledgements}

This research was supported by the Natural Sciences and Engineering Research Council of Canada (NSERC) Discovery Grant Program (P.B.C.), the Canada Research Chairs Program (P.B.C.), the United States Defense Advanced Research Projects Agency ('Topological Excitations in Electronics (TEE)', agreement \#D18AC00011, P.B.C.) and the United States Army Research Office (award no. W911NF-19-1-0211, P.B.C.).

\section{Author contributions}

P.B.C., F.K. and S.S. conceived the idea. K.J., K.R.H. and S.S. performed the measurements. S.S. analysed the data and wrote the first draft of the manuscript. P.B.C. and S.S. supervised the experiments. All authors discussed the results and contributed to the manuscript.

\section{Competing interests}

The authors declare no competing interests.

\section{Additional information}

Supplementary information The online version contains supplementary material available at https://doi.org/10.1038/s41566-021-00832-9.

Correspondence and requests for materials should be addressed to S.S.

Peer review information Nature Photonics thanks Andrea Alu, Andrew Forbes and the other, anonymous, reviewer(s) for their contribution to the peer review of this work.

Reprints and permissions information is available at www.nature.com/reprints. 